УДК 548.0:534

\title{
Influence of Uniaxial Pressure on Elastic Waves Propagation in Piezoelectric Layered Structures "Y-cut langasite/fused silica"
}

Sergey I. Burkov*

Institute of Engineering Physics and Radio Electronics

Siberian Federal University Svobodny, 79, Krasnoyarsk, 660041

Russia

Olga P. Zolotova ${ }^{\dagger}$

Siberian State Aerospace University Krasnoyarskiy Rabochiy, 31, Krasnoyarsk, 660014

Russia

Pavel P. Turchin

Institute of Engineering Physics and Radio Electronics

Siberian Federal University Svobodny, 79, Krasnoyarsk, 660041

Russia

Received 15.09.2014, received in revised form 20.10.2014, accepted 25.11.2014

The study of influence of external uniaxial mechanical stress on the dispersive characteristics and anisotropy of elastic waves in such piezoelectric structures as " $Y$-cut $\mathrm{La}_{3} \mathrm{Ga}_{5} \mathrm{SiO}_{14} /$ fused silica" has been conducted. The results of computer simulation taking into account the linear and nonlinear material constants of materials have been presented. The comparison of elastic wave velocity changes under the influence of an uniaxial stress while a full set of nonlinear material constants of crystalline layer + geometric nonlinearity, or only geometric nonlinearity of the layer induced by the static deformation of a substrate has been fulfilled.

Keywords: piezoelectric layered structure, Rayleigh wave, Love wave, initial stress influence.

\section{Introduction}

Due to extensive use of acousto-electronic devices, such as sensors, filters and frequency transformers in industry and medicine, the study of external static fields influence (especially uniaxial mechanical strain) upon the elastic waves' characteristics in crystals attracts attention of the devices' designers, including those on the basis of the new advanced materials $[1,2]$. Piezoelectric sensors, for example, are widely used for pressures measurement (100-200 MPa/V) at frequencies from $40 \mathrm{kHz}$ to $100 \mathrm{MHz}$ [3].

Mechanical stress significantly changes characteristics of the piezoelectric device due to the changes in both the material properties themselves and the sample's geometrical dimensions.

\footnotetext{
*sburkov@sfu-kras.ru

†dholza@mail.ru

${ }^{\ddagger}$ pturchin@sfu-kras.ru

(c) Siberian Federal University. All rights reserved
} 
When studying or designing the acoustic wave sensor, it is assumed that the piezoelectric device's behavior will be linear. However, if the sensors are exposed to very strong external elastic field, stress amplitude in the piezoelectric material becomes so large that non-linearity, which affects stability and performance of piezoelectric sensors can no longer be ignored [4]. The results of changes in the physical properties of crystal under the uniaxial mechanical pressure influence $[5,6]$ were used to stabilize the resonator's frequency, especially for temperature compensation of the resonators' frequency escape on the basis of quartz crystals and langasite $[7,8]$. The analysis of changes in SAW velocity induced by radial force and torque's application to the resonator on the basis of quartz and langasite crystals was obtained by the authors [9]. It is noted that thirdorder crystal material tensors ignoring leads to considerable error in the resonance frequency drift determination.

The general theory of ground wave propagation in a piezoelectric crystal under conditions of uniaxial mechanical stress, as well as the theory of reflection and refraction of elastic waves at two solid media's interface surface exposed to the external field was developed in the papers $[10,11]$.

Such structures as "layer/substrate" (dielectric or piezoelectric layer of definite thicknesses, placed on a semi-infinite substrate from another material) are widely used for the acoustoelectronic devices development. The authors $[12,13]$ studied the effect of initial stress on Love waves' characteristics in the structures of "piezoelectric layer/isotropic substrate" and "isotropic layer/piezoelectric substrate" types. It is necessary to note that mostly tetragonal, hexagonal and cubic crystals, as well as transversely isotropic medium are considered as piezoelectric medium.

Langasite crystal that belongs to the trigonal class, as well as its isomorphs are widely used for various acousto-electronic devices manufacturing $[14,15]$. The most promising areas of SAW propagation that combines high value of the electromechanical coupling coefficient $\left(K_{2} \sim 0.5 \%\right)$, near zero value temperature coefficient of frequency (TCF) and the minimum value of power flow angle are found for a number of the rotated $Y$-cut langasite $[16,17]$.

When calculating the parameters of a device on the basis of piezostructure under mechanical stress influence, the developers face the following question: whether it is necessary to take into account in calculations set of nonlinear piezoelectric material constants, i.e. how waves' phase velocity changes at that. Unfortunately, for the widely known crystals that are used as piezoelectric films, such ZnO or AlN $[18,19]$, nonlinear material constants are unknown. In this case, valuation of the phase velocity changes under the stress influence can be associated only with the geometric nonlinearity, such as changes in linear dimensions and volume distortion of the sample.

The article is dedicated to the study of the dispersion characteristics and anisotropy of the most important characteristics of the elastic waves in the piezoelectric layered structure, such as phase velocities, electromechanical coupling coefficient (EMCC), and phase velocities control coefficients under conditions of uniaxial pressure. In addition, the main objective of the study is to assess the impact of the material tensors of the third-order crystal on elastic waves' parameters in the $Y$-cut langasite based structures.

\section{The theory of elastic wave propagation in a layered piezoelectric structure under homogeneous uniaxial stress influence}

Suppose the $X_{3}$ axis of orthogonal coordinate system is directed along the outer normal to the surface of the layer, occupying the $0 \leqslant X_{3} \leqslant h$ space, and the $X_{1}$ axis coincides with the 
wave propagation direction, $h$ is the layer thickness. Following the theory developed in [20], let us write the basic equations describing the effects of homogeneous stress and dc electric field simultaneously on the acoustic wave propagation in a piezoelectric medium. As a result of such effects a homogeneous deformation of a crystal should be arisen. Referring to the initial coordinate system, the small-amplitude wave equation of motion, the equation of electrostatics and the state equations for the uniformly deformed acentric crystal, as well as electrostatic equations would take the following form:

$$
\begin{aligned}
& \rho_{0} \ddot{\tilde{U}}_{A}=\tilde{\tau}_{A B, B}+\tilde{U}_{A, P Q} \bar{\tau}_{P Q} ; \\
& \tilde{D}_{M, M}=0 ; \\
& \tilde{\tau}_{A B}=c_{A B C D}^{*} \tilde{\eta}_{C D}-e_{M A B}^{*} \tilde{E}_{M} ; \\
& \tilde{D}_{M}=\varepsilon_{M N}^{*} \tilde{E}_{N}+e_{M A B}^{*} \tilde{\eta}_{A B} .
\end{aligned}
$$

In equation (1) the following values are used: $\rho_{0}$ is the crystal density in the undeformed (initial) state, $\tilde{U}_{A}$ is the vector of dynamic elastic displacement, $\tau_{A B}$ is thermodynamic stress tensor, $\eta_{A B}$ is strain tensor, $E_{M}$ and $D_{M}$ are vectors of electric field and electric displacement, respectively. Here and elsewhere, the time-dependent variables are marked by the "tilde" symbol. The comma after the subscript denotes the spatial derivatives and coordinate Latin indices vary from 1 to 3 . Here and elsewhere, the rule of summation over repeated indices is used. Taking into account only the effect of static uniaxial stress as $\bar{\tau}_{M N}=-\bar{\tau} P_{M} P_{N}\left(P_{M}\right.$ is the unit vector of force direction, and $\bar{\tau}$ is the stress magnitude), the effective elastic, piezoelectric, and dielectric constants which are the linear functions of stress, should be defined as:

$$
\begin{aligned}
& c_{A B K L}^{*}=c_{A B K L}^{E}-c_{A B K L Q R}^{E} s_{Q R M N}^{E} P_{M} P_{N} \bar{\tau} ; \\
& e_{N A B}^{*}=e_{N A B}-e_{N A B K L} s_{K L M N}^{E} P_{M} P_{N} \bar{\tau} ; \\
& \varepsilon_{M N}^{*}=\varepsilon_{M N}^{\eta}-H_{N M A B} s_{A B K L}^{E} P_{K} P_{L} \bar{\tau} .
\end{aligned}
$$

Here, $c_{A B K L}^{E}, e_{N A B}$, and $\varepsilon_{M N}^{\eta}$ are elastic, piezoelectric, and dielectric constants of the second order; $s_{A B K L}^{E}$ is the elastic compliance constant; $c_{A B K L Q R}^{E}, e_{N A B K L}$, and $H_{N M A B}$ are the nonlinear elastic, piezoelectric and electrostriction material tensors.

Substituting into (1) the solutions for the elastic displacements and electric potential in the conventional form of plane monochromatic waves, one can obtain the linearized Green-Christoffel equations with the following components:

$$
\begin{aligned}
& {\left[\Gamma_{B C}(\bar{\tau})-\rho_{0} \omega^{2} \delta_{B C}\right] \alpha_{C}=0} \\
& \Gamma_{B C}=\left[c_{A B C D}^{*}+\left(2 c_{M B F N}^{E} s_{A D C F}^{E}+\delta_{B C} \delta_{A M} \delta_{D N}\right) P_{M} P_{N} \bar{\tau}\right] k_{A} k_{D} ; \\
& \Gamma_{C 4}=e_{P A C}^{*} k_{P} k_{A} ; \quad \Gamma_{4 C}=\Gamma_{C 4}+2 e_{A F D} s_{M N C F}^{E} P_{M} P_{N} \bar{\tau} k_{A} k_{D} ; \quad \Gamma_{44}=-\varepsilon_{P Q}^{*} k_{P} k_{Q} .
\end{aligned}
$$

Here $k_{A}$ is the wave vector components.

In general case, the elastic displacement and electric potential in both media have the form of linear combination of the partial waves such as:

$$
\begin{aligned}
& U_{i}=\sum_{n} a_{n}^{(1,2)} \alpha_{i}^{(n)} \exp \left[i\left(k_{1} x_{1}+k_{3}^{(n)} x_{3}-\omega t\right)\right] \\
& \Phi=\sum_{n} a_{4}^{(1,2)} \alpha_{4}^{(n)} \exp \left[i\left(k_{1} x_{1}+k_{3}^{(n)} x_{3}-\omega t\right)\right] .
\end{aligned}
$$

Here and elsewhere superscripts 1 or 2 denote the substrate and layer terms; $a_{n}$ and $\alpha_{i}$ values are the weight coefficients and partial wave amplitudes respectively. The wave vector component 
$k_{1} \equiv k_{1}^{(n)}=\omega / v$ coincides with the direction of the elastic wave propagation, and the condition $\operatorname{Im}\left(k_{3}^{(n)}\right)<0$ should be satisfied within a substrate, which provides attenuation of the elastic wave below the "layer-substrate" interface.

SAW propagation in a layered structure under the homogeneous stress must comply with the relevant boundary conditions. So, the normal components of the stress tensor should vanish on the free surface of the layer if the pressure is applied along all the directions orthogonal to the free surface. Continuity of tangential component of the electric field should be provided by the continuity of the electric potential at the "layer-vacuum" interface. In addition, at the "layer-substrate" interface when $X_{3}=0$, the equality of normal components of the stress tensor and elastic displacements, as well as continuity of the electric potential must be satisfied. All these requirements can be written as follows:

$$
\begin{aligned}
& \tau_{3 A}^{(2)}=\left.0\right|_{X_{3}=h} ; \quad D_{3}^{(2)}=\left.D^{(v a c)}\right|_{X_{3}=h} ; \quad \varphi^{(2)}=\left.\varphi^{(v a c)}\right|_{X_{3}=h} ; \\
& \tau_{3 A}^{(1)}=\left.\tau_{3 A}^{(2)}\right|_{X_{3}=0} ; \quad D_{3}^{(1)}=\left.D_{3}^{(2)}\right|_{X_{3}=0} ; \quad \varphi^{(1)}=\left.\varphi^{(2)}\right|_{X_{3}=0} ; \quad U_{A}^{(1)}=\left.U_{A}^{(2)}\right|_{X_{3}=0} .
\end{aligned}
$$

Superscripts 1 and 2 denote substrate and layer respectively.

In the case of mechanical stress application orthogonally to the free surface $\left(\vec{P} \| X_{3}\right)$, elastic properties of loading environment should be taken into account. Supposing that uniaxial stress in such geometry is conducted without hard elastic contact with the free surface (e.g. gas environment loading), and for this case mechanical boundary conditions can be written as follows [21]:

$$
\tilde{\tau}_{3 J}+\tilde{U}_{J, K} \bar{\tau}_{3 K}=0 \quad\left(X_{3}=0\right) .
$$

After substituting the solutions (4) in the form of uniform plane waves into boundary conditions (5) and taking into account expressions (1) and (2), one can obtain a system of equations for calculating the parameters of acoustic wave propagation in the layered structures [22]. Calculation of the elastic wave parameters can be fulfilled by the conventional method of partial waves [23]. The condition of vanishing of the boundary conditions determinant allows us to find the phase velocities of the elastic waves.

\section{Dispersive dependences of elastic wave parameters in layered piezoelectric structures}

On the basis of expressions (1)-(6) that describe elastic wave propagation in the layered structure the analysis of changes in characteristics of Rayleigh and Love elastic wave modes due to the change in the effective symmetry of the crystallic piezostructure, exemplified by " $\mathrm{La}_{3} \mathrm{Ga}_{5} \mathrm{SiO}_{14}$ /fused silica" structure at different variants of uniaxial mechanical stress was conducted. Changes of the elastic wave phase velocity in the layered structure under mechanical stress influence would be more convenient to consider on the basis of the elastic wave phase velocity control coefficient that looks as follows:

$$
\alpha_{v}=\frac{1}{v(0)}\left(\frac{\Delta v}{\Delta \bar{\tau}}\right)_{\Delta \bar{\tau} \rightarrow 0} .
$$

The magnitude of the electromechanical coupling coefficient $(E M C C)$ was determined by the following relation:

$$
K^{2}=2 \frac{v-v_{m}}{v},
$$


where $v$ and $v_{m}$ are SAW phase velocities at free or metallized free surface of the piezoelectric layer [24].

Dispersive dependences of the phase velocities, EMCC's, and $\alpha_{v}$ coefficients for Love and Rayleigh waves propagating in the layered structure "[001](010) $\mathrm{La}_{3} \mathrm{Ga}_{5} \mathrm{SiO}_{14} /$ fused silica" (Fig. 1, a) depending on $h \times f$ value ( $h$ is the layer's thickness, and $f$ is the wave frequency) are represented in Fig. 1. This direction was chosen for studing because $Y$-cut of LGS crystal is the most popular for acoustoelectronics devices creation. Material constants' value for linear and nonlinear electromechanical properties were taken for the $\mathrm{La}_{3} \mathrm{Ga}_{5} \mathrm{SiO}_{14}$ (LGS) crystal from [25], and for fused silica - from [26].

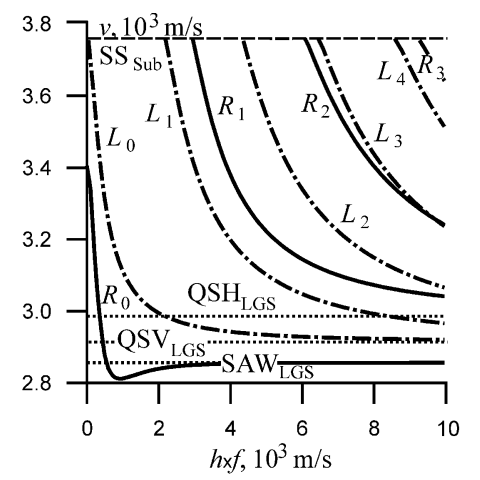

a

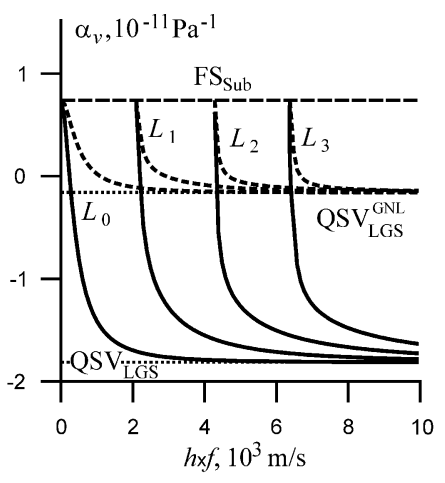

d

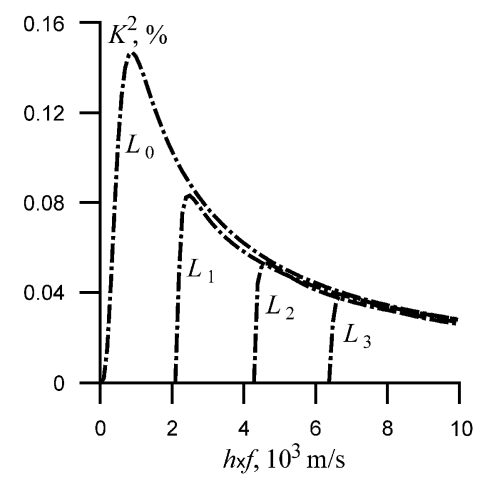

b

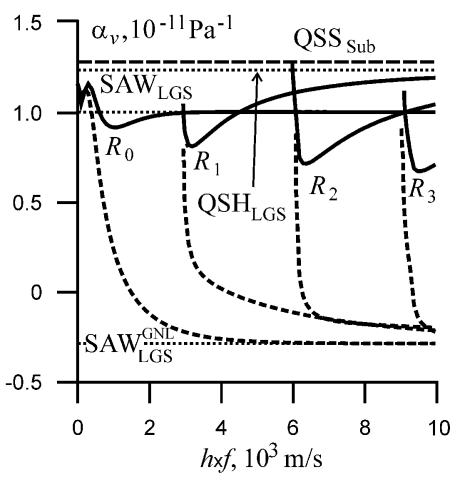

e

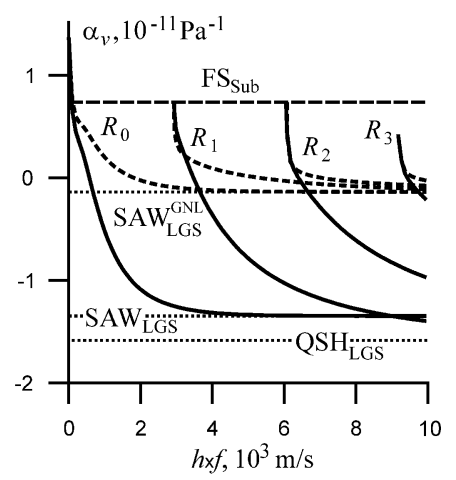

c

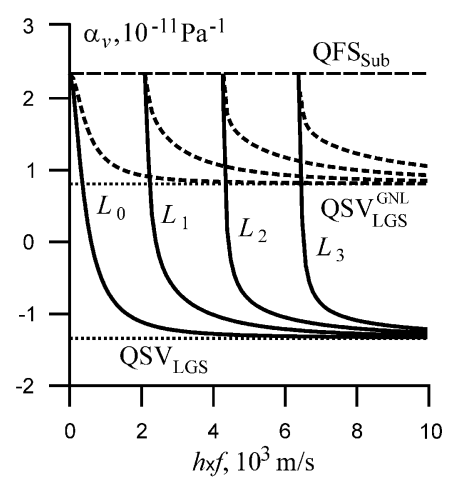

$\mathrm{f}$

Fig. 1. Dispersive dependences of acoustic wave parameters for the layered structure "[001](010) LGS/fused silica": (a) phase velocities; (b) EMCC's at $X_{3}=h ; \alpha_{v}$ coefficients for Rayleigh (c) and Love (d) modes at $\vec{P} \| X_{1} ; \alpha_{v}$ coefficients for Rayleigh (e) and Love (f) waves at $\vec{P} \| X_{2}$.

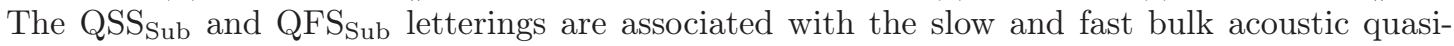
shear waves in the substrate; Rayleigh wave in the layer is marked by the $\mathrm{SAW}_{\mathrm{LGS}}$ letterings. Solid lines are associated with FSN option, and dashed lines - with GNL option. Curves $R_{i}$ and $L_{i}$ are associated with Rayleigh and Love modes of several orders

When acoustic waves propagate along [001] direction of the LGS crystal plane (010), the two-fold symmetry axis lies orthogonally to the sagittal plane, thus, in this case pure modes of Love and Rayleigh waves propagate, as in this case the Green-Christoffel tensor (3) is divided into two independent parts.

For LGS single crystal in this direction Rayleigh surface wave (SAW) can propagate, but 
not Bleustein-Gulyaev wave (BGW). In [001](010) plane of the LGS crystal SAW phase velocity equals $2855.74 \mathrm{~m} / \mathrm{s}$. Volume shift elastic waves are degenerated and phase velocity of FS wave is equal to $3052.1 \mathrm{~m} / \mathrm{s}$.

It should be noted that in the layered structure "[001](010) LGS/fused silica" bulk acoustic waves can transform into non-dispersive surface elastic waves, which can be classified as "Anisimkin Jr. mode" (AN) $[27,28]$. The AN mode has such basic properties as dominant longitudinal displacement $\left(U_{1} \gg U_{2}, U_{3} ; U_{1} \approx\right.$ constant within the plate or layer), or a dominant shear displacement for the shear-horizontal QSH $\left(U_{2} \gg U_{1}, U_{3}\right)$, and the shear-vertical QSV $\left(U_{3} \gg U_{1}, U_{2}\right)$ waves [29]. Moreover, the approximate equality of phase velocities for the BAW and AN modes should be observed. In the case under consideration QFS wave transforms into QSH mode, but QSS wave transforms into QSV mode (Fig. 1). It should be noted that transformation of bulk waves into AN modes in this case occurs linearly, i.e. without external influence.

The $h \times f$ change was from 0 up to $10000 \mathrm{~m} / \mathrm{s}$. Variation range of Love wave phase velocities is between the value of shear wave velocity for fused silica and slow quasi-shear wave (QSV) phase velocity for LGS (2913.89 m/s) (Fig. 1, a). The dispersive curve for the $R_{0}$ zero-mode Rayleigh wave starts at Rayleigh phase velocity for fused silica $(3405.23 \mathrm{~m} / \mathrm{s})$, passes through a minimum at $h \times f=900 \mathrm{~m} / \mathrm{s}$, and gradually increases with $h \times f$. The value increases, then coming up to the phase velocity of Rayleigh SAW for langasite. Phase velocities for higher order modes of Rayleigh wave are located between the shear wave velocity for fused silica and the velocity for the fast quasi-shear wave (QSH) for langasite $(2985.56 \mathrm{~m} / \mathrm{s})$. Only Love wave has low piezoelectric activity. The EMCC calculation was performed under condition of top metallization on the piezoelectric layer $\left(X_{3}=h\right)$, and the maximal value of $K^{2}=0.15 \%$ at $h \times f=900 \mathrm{~m} / \mathrm{s}$ is observed for the $L_{0}$ mode of Love wave (Fig. $1, \mathrm{~b}$ ).

Application of external mechanical stress with effective symmetry $\infty / \mathrm{mmm}$ along elastic wave propagation direction $\left(\vec{P}\left\|X_{2}\right\|[100]\right)$ according to the Curie symmetry principle, decreases LGS initial crystal symmetry to monoclinous. As a result, modification of the crystal material constants take place:

$$
\begin{aligned}
& c_{22}^{*}=c_{11}^{E}-\left(c_{222} s_{12}+c_{113} s_{13}+c_{224} s_{14}\right) \bar{\tau} \\
& c_{24}^{*}=c_{42}^{*}=-c_{14}^{E}-\left(c_{124} s_{11}+c_{224} s_{12}-c_{134} s_{13}+c_{155} s_{14}\right) \bar{\tau} ; \\
& c_{23}^{*}=c_{32}^{*}=c_{13}^{E}-\left[c_{123} s_{11}+c_{113} s_{12}+c_{133} s_{13}-c_{134} s_{14}\right] \bar{\tau} ; \\
& c_{33}^{*}=c_{33}^{E}-\left[c_{133}\left(s_{11}+s_{12}\right)+c_{333} s_{13}\right] \bar{\tau} ; \\
& c_{34}^{*}=c_{43}^{*}=-\left[c_{134}\left(s_{11}-s_{12}\right)+c_{344} s_{14}\right] \bar{\tau} ; \\
& c_{44}^{*}=c_{44}^{E}-\left(c_{144} s_{11}+c_{155} s_{12}+c_{344} s_{13}+c_{444} s_{14}\right) \bar{\tau} ; \\
& c_{55}^{*}=c_{44}^{E}-\left(c_{155} s_{11}+c_{144} s_{12}+c_{344} s_{13}-c_{444} s_{14}\right) \bar{\tau} ; \\
& c_{56}^{*}=c_{65}^{*}=c_{14}^{E}-\left(c_{156} s_{11}+c_{256} s_{12}+c_{134} s_{13}+c_{456} s_{14}\right) \bar{\tau} ; \\
& c_{66}^{*}=c_{66}^{E}-\left(c_{166} s_{11}+c_{266} s_{12}+c_{366} s_{13}+c_{124} s_{14}\right) \bar{\tau} ; \\
& e_{25}^{*}=-e_{14}+\left(e_{124} s_{11}+e_{114} s_{12}+e_{134} s_{13}-e_{144} s_{14}\right) \bar{\tau} ; \\
& e_{26}^{*}=-e_{11}-\left(e_{216} s_{11}+e_{226} s_{12}-e_{113} s_{13}-e_{156} s_{14}\right) \bar{\tau} ; \\
& e_{35}^{*}=-\left[e_{315}\left(s_{11}-s_{12}\right)\right] \bar{\tau} ; \\
& e_{36}^{*}=e_{315} s_{14} \bar{\tau} ; \\
& e_{216}=\frac{1}{4}\left(e_{111}+3 e_{122}\right) ; \quad e_{226}=-\frac{1}{4}\left(3 e_{111}+e_{122}\right) ; \\
& \varepsilon_{11}^{*}=\varepsilon_{11}^{\eta}-\left(H_{11} s_{11}+H_{12} s_{12}+H_{13} s_{13}+H_{14} s_{14}\right) \bar{\tau} ; \\
& \varepsilon_{33}^{*}=\varepsilon_{33}^{\eta}-\left[H_{31}\left(s_{11}+s_{12}\right)+H_{33} s_{13}\right] \bar{\tau}
\end{aligned}
$$


Thus, the symmetry axis of the second order crystal orthogonally to the sagittal plane, and, as it was mentioned above, in this case Green-Christoffel tensor (3) is divided into two independent parts and its components, taking into account static Green's tensor $\bar{\eta}_{A B}=\delta_{A B}+2 s_{A B C D} \bar{\tau}_{C D}$, look as follows:

$$
\begin{aligned}
\Gamma_{11} & =\left[c_{22}^{*}-2\left(c_{11} s_{12}-c_{14} s_{14}\right) \bar{\tau}\right] k_{1}^{2}+\left[2 c_{24}^{*}+2\left(2 c_{14} s_{12}-\left(c_{13}+c_{44}\right) s_{14}\right) \bar{\tau}\right] k_{1} k_{3}+ \\
& +\left[c_{44}^{*}-2 c_{44} s_{12} \bar{\tau}\right] k_{3}^{2} ; \\
\Gamma_{13} & =\left[c_{24}^{*}+2\left(c_{14} s_{13}-c_{11} s_{14}\right) \bar{\tau}\right] k_{1}^{2}+\left[c_{23}^{*}+c_{44}^{*}+2\left(2 c_{14} s_{14}-\left(c_{13}+c_{44}\right) s_{13}\right) \bar{\tau}\right] k_{1} k_{3}+ \\
& +\left[c_{34}^{*}-2 c_{44} s_{14} \bar{\tau}\right] k_{3}^{2} ; \\
\Gamma_{31} & =\left[c_{24}^{*}+2\left(c_{14} s_{12}-c_{44} s_{14}\right) \bar{\tau}\right] k_{1}^{2}+\left[c_{23}^{*}+c_{44}^{*}-2\left(c_{13}+c_{44}\right) s_{12} \bar{\tau}\right] k_{1} k_{3}+ \\
& +\left[c_{34}^{*}-2 c_{33} s_{14} \bar{\tau}\right] k_{3}^{2} ; \\
\Gamma_{22} & =\left[c_{66}^{*}-2 c_{66} s_{11} \bar{\tau}\right] k_{1}^{2}+\left[2 c_{56}^{*}-4 c_{14} s_{11} \bar{\tau}\right] k_{1} k_{3}+\left[c_{55}^{*}-2 c_{44} s_{11} \bar{\tau}\right] k_{3}^{2} ; \\
\Gamma_{33} & =\left[c_{44}^{*}-2\left(c_{44} s_{13}-c_{14} s_{14}\right) \bar{\tau}\right] k_{1}^{2}+\left[2 c_{34}^{*}-2\left(c_{13}+c_{44}\right) s_{14} \bar{\tau}\right] k_{1} k_{3}+\left[c_{33}^{*}-2 c_{33} s_{13} \bar{\tau}\right] k_{3}^{2} ; \\
\Gamma_{24} & =e_{26}^{*} k_{1}^{2}+\left[e_{25}^{*}+e_{36}^{*}\right] k_{1} k_{3}+e_{35}^{*} k_{3}^{2} ; \\
\Gamma_{42} & =\left[e_{26}^{*}+2 e_{11} s_{11} \bar{\tau}\right] k_{1}^{2}+\left[e_{25}^{*}+e_{36}^{*}+2 e_{14} s_{11} \bar{\tau}\right] k_{1} k_{3}+e_{35}^{*} k_{3}^{2} ; \\
\Gamma_{44} & =-\left[\varepsilon_{11}^{*} k_{1}^{2}+\varepsilon_{33}^{*} k_{3}^{2}\right] .
\end{aligned}
$$

It should be noted that in this case strong geometrical nonlinearity takes place, i.e. geometric distortion of a layer, and, correspondingly, Green-Christoffel tensor (10) becomes asymmetric, particularly the value of additional parts under stress influence at $\Gamma_{13}$ and $\Gamma_{31}$ components differs more than twice.

Under uniaxial mechanical stress application to fused silica that represent isotropic medium, the initial symmetry also decreases to hexagonal with preferential direction along mechanical stress application and indication of new effective elastic moduli. In particular, when application of uniaxial homogeneous stress coincides with the direction of the elastic wave propagation, effective elastic moduli can be represented in the following form:

$$
\begin{aligned}
& c_{11}^{*}=c_{11}-\left(c_{111} s_{11}+2 c_{112} s_{12}\right) \bar{\tau} \\
& c_{22}^{*}=c_{33}^{*}=c_{11}-\left[c_{112} s_{11}+\left(c_{111}+c_{112}\right) s_{12}\right] \bar{\tau} ; \\
& c_{12}^{*}=c_{13}^{*}=c_{12}-\left[c_{112} s_{11}+\left(c_{112}+c_{123}\right) s_{12}\right] \bar{\tau} ; \\
& c_{23}^{*}=c_{12}-\left(c_{123} s_{11}+2 c_{112} s_{12}\right) \bar{\tau} ; \\
& c_{44}^{*}=c_{44}-\left(c_{144} s_{11}+2 c_{155} s_{12}\right) \bar{\tau} ; \\
& c_{55}^{*}=c_{66}^{*}=c_{44}-\left[c_{155} s_{11}+\left(c_{144}+c_{155}\right) s_{12}\right] \bar{\tau} ; \\
& c_{14}^{*}=c_{15}^{*}=c_{16}^{*}=c_{24}^{*}=c_{25}^{*}=c_{26}^{*}=c_{34}^{*}=c_{35}^{*}=c_{36}^{*}=c_{45}^{*}=c_{46}^{*}=c_{56}^{*}=0 ; \\
& c_{144}=\frac{1}{2}\left(c_{112}-c_{123}\right) ; \quad c_{155}=\frac{1}{4}\left(c_{111}-c_{112}\right) .
\end{aligned}
$$

As a result, all the elastic moduli depend on the mechanical stress value, and some of them were changed in accordance with the requirements for isotropic media. It is easy to obtain that the relation $c_{44}^{*}=\frac{1}{2}\left(c_{22}^{*}-c_{23}^{*}\right)$ which is written for special orientation of axes is fulfilled for the point group symmetry of $\infty / m$ symmetry. In such media at variation of the phase velocities values of longitudinal and shear waves, degeneration of the shire wave retains. But under conditions of SAW propagation in the directions, orthogonal to the applied external pressure, degeneration of shear wave takes place, but, at that, phase velocities of longitudinal and shear waves remain isotopic. 
Let's consider the mechanical stress influence on the layered structure, taking into account only static deformation of a layer without physical nonlinearity. In this case, effective elastic moduli (9) are equal to the corresponding linear moduli, but in Green-Christoffel tensor (10) components still contain the terms, defined by static Green's tensor, for instance: $\Gamma_{22}=\left[c_{66}-2 c_{66} s_{11} \bar{\tau}\right] k_{1}^{2}+\left[2 c_{14}-4 c_{14} s_{11} \bar{\tau}\right] k_{1} k_{3}+\left[c_{44}-2 c_{44} s_{11} \bar{\tau}\right] k_{3}^{2}$.

In Fig. 1 dispersion characteristic of Rayleigh and Love waves' $\alpha_{v}$ cooefficients in the structure "[001](010) LGS/fused silica" are represented. There are $\alpha_{v}$ control coefficients for Rayleigh and Love waves at $\vec{P} \| X_{1}$ and $\vec{P} \| X_{2}$ (Fig. 1, c-f). Solid lines are associated with $\alpha_{v}$ values obtained by uniaxial stress application taking into account the Full Set of Nonlinearities both for the layer and for the substrate. Such option will be futher designated as FSN. Dashed lines show the values of the control coefficients, calculated taking into account only static deformation of the layer - Geometric Nonlinearity of the Layer, excluding nonlinear material constants of LGS. Such option will be futher designated as GNL. But in the last case uniaxial mechanical stress application for the substrate was taken into account with the full set of nonlinearities of the crystal media.

The $\alpha_{v}$ coefficients for quasi-shear acoustic wave (FS) and for Rayleigh wave in the LGS single crystal, calculated according to the FSN option, are equal to $-1.63 \cdot 10^{-11}$ and $-1.35 \cdot 10^{-11} \mathrm{~Pa}^{-1}$ respectively. The same coefficients calculated according to the GNL parametres have magnitudes $-1.6 \cdot 10^{-12}$ and $-1.37 \cdot 10^{-12} \mathrm{~Pa}^{-1}$ respectively. Shear bulk wave in fused silica, as it was noted above, retains the degeneracy.

Values of the phase velocities of elastic waves in a layered structure, as a rule, are in the range from phase shear wave velocity in the substrate up to the value of SAW velocity for mode $R_{0}$ and up to a value of the QSH wave velocity for Rayleigh waves' modes of higher order, and up to the value of the QSV wave velocity for the elastic waves' modes with transverse horizontal polarization ( $\mathrm{SH}$-waves).

The behavior of dispersive dependences for the $\alpha_{v}$ coefficients for Rayleigh and Love elastic waves' modes under the condition external stress application along the direction of wave propagation $\vec{P} \| X_{1}$ is qualitatively similar for both variants of calculation (Figs. 1c and 1d). Values of the $\alpha_{v}$ coefficients at the calculations in the GNL mode are less for the first and zero Rayleigh wave mode, as a rule $\alpha_{v}=-1.37 \cdot 10^{-12} \mathrm{~Pa}^{-1}$ for the $\mathrm{SAW}$ LGS and for Rayleigh waves modes of the higher order aim for value $\alpha_{v}=-1.57 \cdot 10^{-12} \mathrm{~Pa}^{-1}$ wave $\mathrm{QSH}_{\mathrm{LGS}}^{\mathrm{GNL}}$. The similar control coefficients values for Love waves modes, $\alpha_{v}=-1.6 \cdot 10^{-12} \mathrm{~Pa}^{-1}$ aim for the value QSV GGL .

Under application of mechanical pressure along the axis of the second crystal, i.e. orthogonally to the sagittal plane $\left(\vec{P} \| X_{2}\right)$, the shear wave degeneration dismissal in fused silica takes place, i.e. splitting of shear wave to fast and slow waves for which $\alpha_{v}$ coefficients are equal to the $2.35 \cdot 10^{-11}$ and $1.28 \cdot 10^{-11} \mathrm{~Pa}^{-1}$ respectively. In this case, also, reduction of the effective LGS crystal symmetry to the monocline takes place, and the $\alpha_{v}$ coefficients for the fast and slow shear waves in LGS single crystal are equal to $1.2 \cdot 10^{-11}$ and $-6.41 \cdot 10^{-12} \mathrm{~Pa}^{-1}$ respectively. When the $h \times f$ parameter increases, the $\alpha_{v}$ coefficients for zero Rayleigh mode are equal to $\alpha_{v}=$ $1.0 \cdot 10^{-11} \mathrm{~Pa}^{-1}$ for SAW in $\alpha_{v}$ crystal for Love modes smoothly change from the values observed in fused silica for the fast shear wave $\left(\alpha_{v}=2.35 \cdot 10^{-11} \mathrm{~Pa}^{-1}\right)$ up to $\alpha_{v}=-1.34 \cdot 10^{-11} \mathrm{~Pa}^{-1}$ for QSV wave (Fig. 1, f).

If mechanical pressure is applied along the axis of the second, orthogonally to the sagittal plane, and only geometric nonlinearity is taken into account (GNL option), the $\alpha_{v}$ coefficients for the modes Love elastic waves change as well as in the above case but the limit value for $\mathrm{QSV}_{\mathrm{LGS}}^{\mathrm{GNL}}$ is equal to $8.1 \cdot 10^{-12} \mathrm{~Pa}^{-1}$ (Fig. 1, f). It should be noted, that variations of the $\alpha_{v}$ 
coefficients for Rayleigh wave's modes differ from the similar ones for SH-modes, as a consequence of strong geometrical nonlinearity (Fig. 1, e). Values of the $\alpha_{v}$ coefficients asymptotically aim $R_{0}$ wave modes to $\alpha_{v}=-2.85 \cdot 10^{-12} \mathrm{~Pa}^{-1}$ for $\mathrm{SAW}_{\mathrm{LGS}}^{\mathrm{GNL}}$ and for the higher order modes, $\alpha_{v}=-2.75 \cdot 10^{-12} \mathrm{~Pa}^{-1}$ for $\mathrm{QSH}_{\mathrm{LGS}}^{\mathrm{GNL}}$. Thus, if only static deformation measurement in this case leads to "decrease" of the elastic wave phase velocity value, than measurement of all the nonlinear material constants lead to "increase".

The results of this direction analysis are similar to those, received earlier for "[010](001) $\mathrm{La}_{3} \mathrm{Ga}_{5} \mathrm{SiO}_{14} /$ fused silica" structure in [22]. Consequently, ignoring nonlinear material tensors of LGS crystal during calculations also leads to significant error in calculations of Rayleigh and Love elastic waves' velocities.

Dispersive dependences of the phase velocities, EMCC's, and $\alpha_{v}$ coefficients for Rayleigh and Love waves in the " $\left(0^{\circ}, 138.5^{\circ}, 26.7^{\circ}\right) \mathrm{La}_{3} \mathrm{Ga}_{5} \mathrm{SiO}_{14} /$ fused silica" structure, determined by Euler angles $\left(0^{\circ}, 138.5^{\circ}, 26.7^{\circ}\right)$, taking into account nonlinear material LGS tensors are presented in Fig. 2. Such direction in langasite crystal is of interest for acoustoelectronics devices creation [17] due to the presence of SAW thermostable properties, with relatively high EMCC value and zero angle of energy flow deviation $[16,30]$.

In the layered structure " $\left(0^{\circ}, 138.5^{\circ}, 26.7^{\circ}\right) \mathrm{La}_{3} \mathrm{Ga}_{5} \mathrm{SiO}_{14} /$ fused silica" transformation of bulk acoustic waves in LGS crystal into the non-dispersive elastic wave of "Anisimkin Jr. mode" type (QSH and QSV), even in the absence of mechanical stress also takes place.

Dispersion dependence of zero-mode Rayleigh wave velocity starts from Rayleigh SAW in fused silica $(3405.23 \mathrm{~m} / \mathrm{s})$, passes through a minimum at $h \times f=900 \mathrm{~m} / \mathrm{s}$, and then with the increase $h \times f$ gradually increases to SAW value in langasite single crystal $(2735.96 \mathrm{~m} / \mathrm{s})$ and further with $h \times f=2500 \mathrm{~m} / \mathrm{s}$ is practically non-dispersive. The interval of phase velocities chages of all the other modes is between the values of shear waves phase velocities of fused silica and QSV slow shear wave of LGS crystal (Fig. 2, a). Both Rayleigh and Love modes have piezoelectric activity, and maximal value $K^{2}=0.54 \%$ at $h \times f=600 \mathrm{~m} / \mathrm{s}$ is obseved for the $L_{0}$ mode of Love wave (Fig. 2, b).

Fig. 2, c and 2, d show the $\alpha_{v}$ coefficients for Rayleigh and Love waves under the application of uniaxial homogeneous stress along the axis of wave propagation $\vec{P} \| X_{1}$, and the dispersive dependences calculations are qualitatively similar for the both variants (FSN and GNL).

The values of $\alpha_{v}$ coefficients for the fundamental Rayleigh mode beginning with $h \times f=$ $2500 \mathrm{~m} / \mathrm{s}$ are constant and equal $\alpha_{v}=-1.99 \cdot 10^{-11} \mathrm{~Pa}^{-1}$ of SAW in LGS single crystal. The values of $\alpha_{v}$ coefficients for Love wave mode, as well as Rayleigh mode of the highest order decrease from the value of silica's shear wave $\left(\alpha_{v}=7.4 \cdot 10^{-12} \mathrm{~Pa}^{-1}\right)$ and strive down to $\alpha_{v}=-2.026 \cdot 10^{-11} \mathrm{~Pa}^{-1}$ slow shear LGS, in contrast to the case of clear mode propagation. It should be noted that all the elastic waves' modes in this case are weak dispersive, for example, for $R_{1}$ mode $\alpha_{v}$ coefficients also with $h \times f=5000 \mathrm{~m} / \mathrm{s}$ practically do not change $\left(\alpha_{v}=-2.026\right.$. $\left.10^{-11} \mathrm{~Pa}^{-1}\right)$. It is notisable, that the $\alpha_{v}$ coefficients values for SAW and QSV elastic waves are almost identical.

Values of the $\alpha_{v}$ coefficients for Rayleigh modes under condition of GNL option are three times less then usual, and asymptotically tend to $\alpha_{v}=-1.78 \cdot 10^{-12} \mathrm{~Pa}^{-1}$ for the $\mathrm{SAW}_{\mathrm{LGS}}^{\mathrm{GNL}}$. Similar values of the control coefficients for Love modes tend to $\alpha_{v}=-2.2 \cdot 10^{-12} \mathrm{~Pa}^{-1}$ for $\mathrm{QSV}_{\mathrm{LGS}}^{\mathrm{GN}}$.

Figs. 2, e and 2, f show the $\alpha_{v}$ coefficients for Love and Rayleigh waves when uniaxial homogeneous stress is applied orthogonally to the sagittal plane $\vec{P} \| X_{2}$. The $\alpha_{v}$ coefficient value is constant only for the fundamental $R_{0}$ mode, beginning with $h \times f=3000 \mathrm{~m} / \mathrm{s}$, but an oscillating 


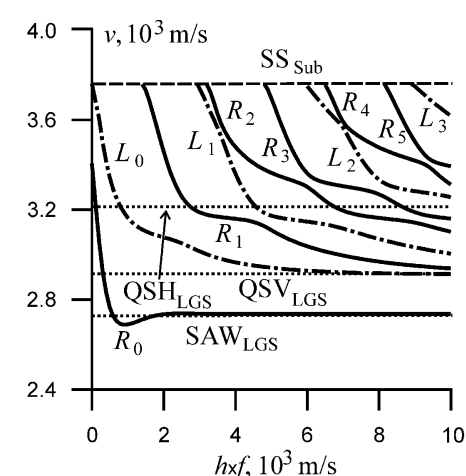

a

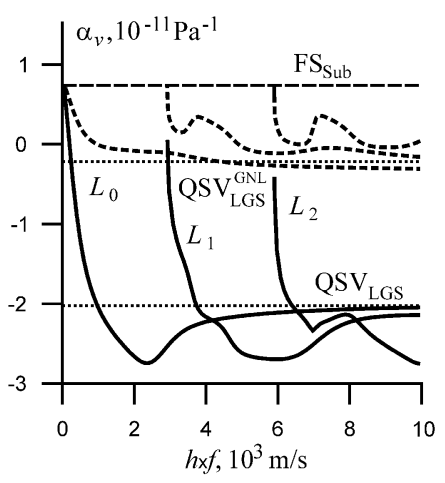

$\mathrm{d}$

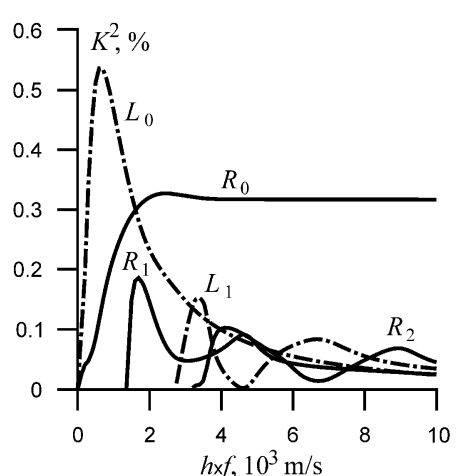

b

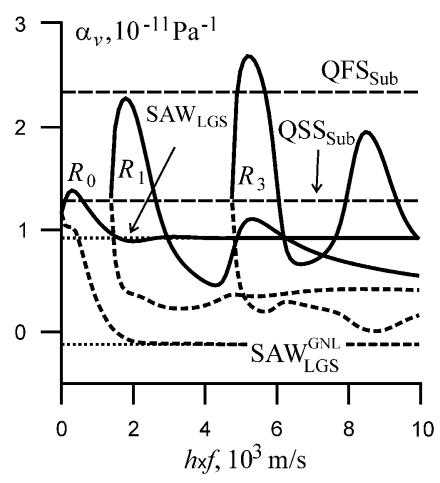

$\mathrm{e}$

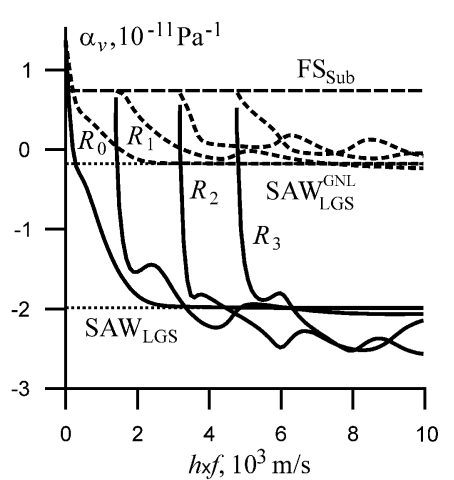

$\mathrm{c}$

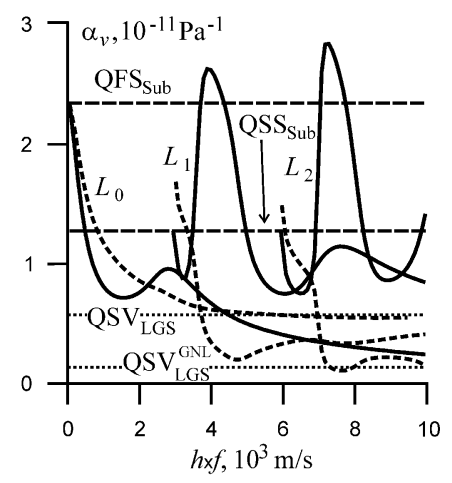

f

Fig. 2. Dispersion of acoustic wave parameters in the " $\left(0^{\circ}, 138.5^{\circ}, 26.7^{\circ}\right)$ LGS/fused silica" layered structure: (a) phase velocities; (b) EMCCs at $X_{3}=h ; \alpha_{v}$ coefficients for Rayleigh (c) and Love (d) modes at $\vec{P} \| X_{1} ; \alpha_{v}$ coefficients for Rayleigh (e) and Love (f) modes at $\vec{P} \| X_{2}$. Solid lines are associated with FSN option, and dashed lines - with GNL option

behavior of the $\alpha_{v}$ coefficients for all the other elastic waves' modes is observed. The $\alpha_{v}$ coefficients for all the other elastic wave modes start from $\alpha_{v}=1.28 \cdot 10^{-11} \mathrm{~Pa}^{-1}$ of QSS wave for fused silica.

If mechanical pressure is applied orthogonally to the sagittal plane, i.e. $\vec{P} \| X_{2}$ at the GNL calculation option, the $\alpha_{v}$ coefficients values for Rayleigh and Love modes differ slightly. It should be noted, that in this case, $\alpha_{v}$ coefficients for all the modes have the opposite sign for FSN and GNL options.

Values of $\alpha_{v}$ coefficients asymptotically tend to $\alpha_{v}=-1.21 \cdot 10^{-12} \mathrm{~Pa}^{-1}$ for $\mathrm{SAW}_{\mathrm{LGS}}^{\mathrm{GNL}}$. Note that if only static deformation is taken into account there is "decreasing" effect of wave's phase velocity, but in the case when physical and geometrical nonlinearities were involved, the "increase" of wave's phase velocity takes place $\left(\alpha_{v}=9.18 \cdot 10^{-12} \mathrm{~Pa}^{-1}\right)$. Consideration of nonlinear material tensors of LGS crystal leads to increase of $\alpha_{v}$ coefficient for $\sim 1 \cdot 10^{-11} \mathrm{~Pa}^{-1}$ for the fundamental $R_{0}$ Rayleigh mode, in comparision with GNL option. The difference between FSN and GNL calculation options is more significant for the higher order Rayleigh modes (Fig. 2, e), as well as for Love modes. Thus, behavior variations of $\alpha_{v}$ coefficients for Rayleigh wave's modes significantly differ from SH mode as a consequence of strong geometrical nonlinearity. It should be noted that 
$\alpha_{v}$ coefficients for Love waves' modes change as in above mentioned case, but the limit value for $\mathrm{QSV}_{\mathrm{LGS}}^{\mathrm{GNL}}$ wave is equal to $1.34 \cdot 10^{-12} \mathrm{~Pa}^{-1}$ (Fig. 2, f).

\section{Anisotropy of elastic waves propagating in $\mathrm{Y}$-cut LGS/fused silica layered structure}

Fig. 3 shows anisotropy of the basic characteristics of the principal (zero) and the first mode of Rayleigh and Love acoustic waves in the structure "(010) LGS/fused silica" ( $Y$-cut) at different types of uniaxial mechanical stress applications for the three values of $h \times f=1000,3000,5000 \mathrm{~m} / \mathrm{s}$ parameter. Angle $\psi$ was measured from the direction [100]. The elastic waves' phase velocities are in the range between SAW velocity in langasite crystal and shear wave velocity in fused silica (Fig. 3, a). The peculiarity of the elastic wave propagation in this structure is that the bulk shear LGS waves are transformed into non dispersive surface mode of "Anisimkin Jr. mode" (QSH and QSV) wave in almost all the directions.

In Fig. 3, b, c, d and e control coefficients of Rayleigh and Love elastic waves' $\alpha_{v}$ modes under condition of external mechanical stress application along the direction of the wave propagation $\left(P \| X_{1}\right)$ are represented. Influence of external mechanical stress on QSS and QFS bulk shear waves' phase velocity, as well as in the options studied above, in FSN and GNL option modes acts in different directions, i.e., taking into account nonlinear material LGS tensors when calculating, almost completely compensates geometric nonlinearity of the sample due to external stress application. However, the effect of external mechanical stress on Rayleigh and Love elastic waves' phase velocity in FSN and GNL option modes varies depending on of $h \times f$ values. In particular, $\alpha_{v}$ coefficient for the fundamental $R_{0}$ mode in the direction at an angle $\psi=45^{\circ}$ is equal to $-1.45 \cdot 10^{-11} \mathrm{~Pa}^{-1}$ and $2.21 \cdot 10^{-12} \mathrm{~Pa}^{-1}$ when $h \times f=1000 \mathrm{~m} / \mathrm{s}$ in FSN and GNL option modes and $-2.86 \cdot 10^{-11} \mathrm{~Pa}^{-1},-1.77 \cdot 10^{-13} \mathrm{~Pa}^{-1}$ when $h \times f=5000 \mathrm{~m} / \mathrm{s}$ respectively. For the first mode of Love wave $L_{1}$ at $h \times f=3000 \mathrm{~m} / \mathrm{s}$ consideration of nonlinear material tensors LGS (FSN) changes $\alpha_{v}$ coefficient value only numerically, while the character of $\alpha_{v}$ anisotropy is qualitatively similar for the both modes of calculation (Fig. 3, d, e). The highest value of $\alpha_{v}$ control coefficients in the case of external mechanical stress application $\vec{P} \| X_{1}$ is observed for $R_{0}$ mode at $h \times f=5000 \mathrm{~m} / \mathrm{s}$ and $\psi=58^{\circ}$ and constitutes $-3.13 \cdot 10^{-11} \mathrm{~Pa}^{-1}$, which is close to SAW value at this point $-3.18 \cdot 10^{-11} \mathrm{~Pa}^{-1}$ (Fig. 3 , b).

When external mechanical stress is applied orthogonally to the sagittal plane, i.e. $\vec{P} \| X_{2}$, maximum $\alpha_{v}$ value also belongs to $R_{0}$ mode and constitutes $2.03 \cdot 10^{-11} \mathrm{~Pa}^{-1}$ when $h \times f=$ $5000 \mathrm{~m} / \mathrm{s}$ and $\psi=60^{\circ}$, which is close to SAW value at this point $\alpha_{v}=2.06 \cdot 10^{-11} \mathrm{~Pa}^{-1}$ (Fig. 3, f). Note that $\alpha_{v}$ values for all the modes' types taking into account FSN option in both cases of stress application have the same order of magnitude $\left(10^{-11} \mathrm{~Pa}^{-1}\right)$. The $\alpha_{v}$ values taking into account GNL option in both cases of stress application also have the same order of magnitude $\left(10^{-12} \mathrm{~Pa}^{-1}\right)$, but differ at $\vec{P} \| X_{1}$ и $\vec{P} \| X_{2}$ for 5 times.

\section{Conclusion}

Basic equations and boundary conditions describing acoustic waves' propagation in piezoelectric layered structures under condition of uniaxial mechanical pressure application are derived. Dispersive character of acoustic modes in piezoelectric layered structures " $Y$-cut LGS/fused silica" due to changes in the crystals' effective symmetry under condition of external mechanical 


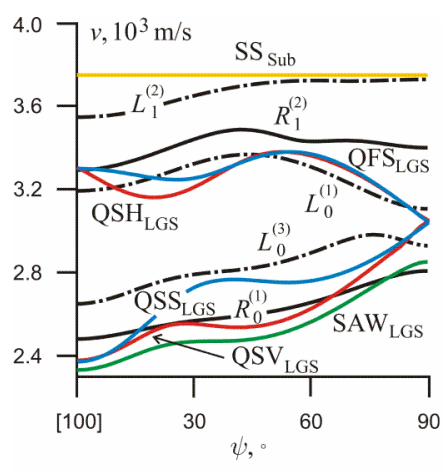

a
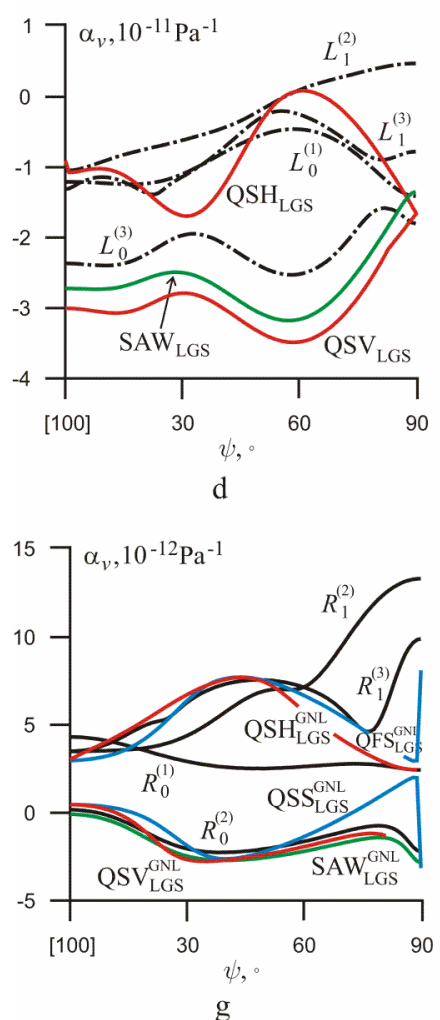

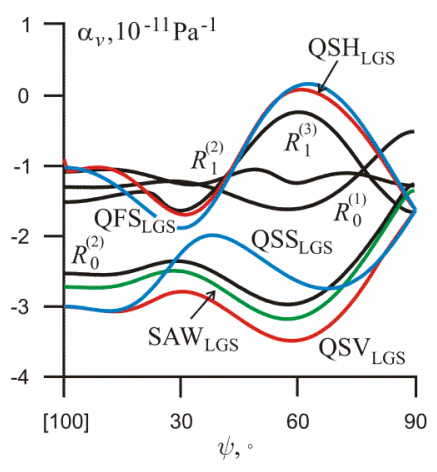

b
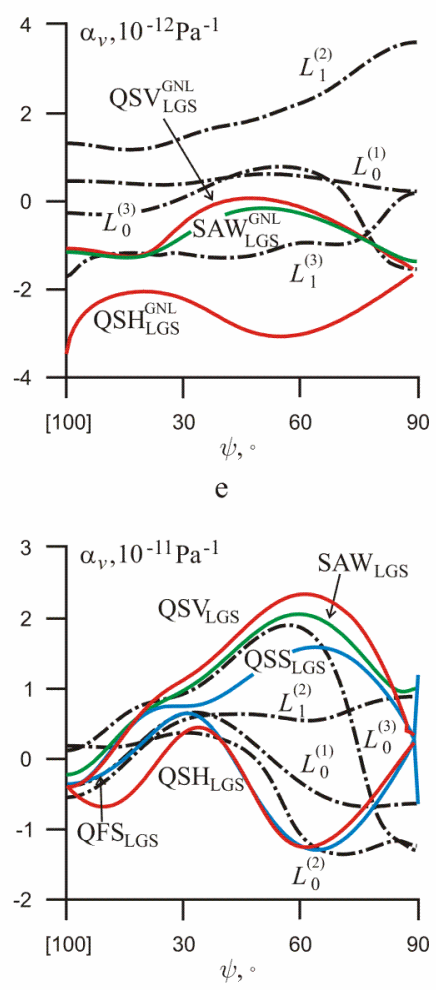

$\mathrm{h}$

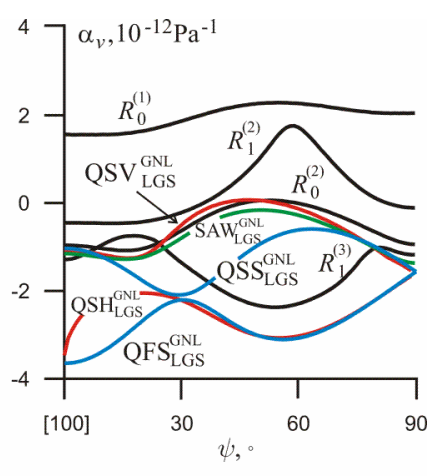

c
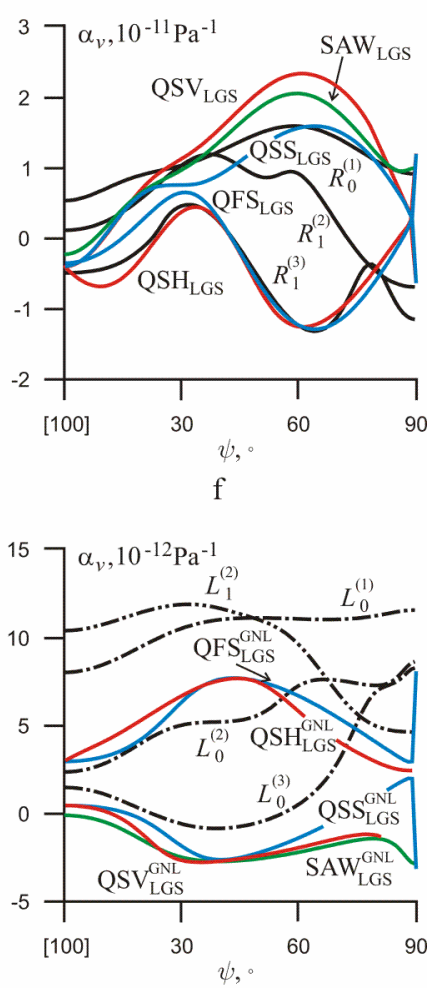

i

Fig. 3. Anisotropy of acoustic wave parameters in the "(010) LGS/fused silica" layered structure for $h \times f(\mathrm{~m} / \mathrm{s})(1)-1000 ;(2)-3000 ;(3)$ - 5000: (a) phase velocities; $\alpha_{v}$ coefficients for Rayleigh (b, c) and Love (d, e) modes at $\vec{P} \| X_{1} ; \alpha_{v}$ coefficients for Rayleigh (f, g) and Love (h, i) modes at $\vec{P} \| X_{2}$

stress application was thoroughly analyzed. Dispersion characteristic of phase velocities, EMCC, control coefficients as $h \times f$ parameter functions are calculated. Valuation of the contribution of nonlinear material tensors of the third-order crystal and static deformation in the layer in changing elastic waves' characteristics due to external mechanical stress application was carries out. It was noted that valuation of material tensors of the third-order crystal increases phase velocities' control coefficients, as a rule, for more than an order, even in the directions where 
mechanical stress influence is minimal. It is important to note that mainly, in the structures under study, static stress effect and valuation of material tensors of the third-order crystal affect phase velocity value oppositely, i.e. in this case, $\alpha_{v}$ coefficients for some modes have the opposite sign for FSN and GNL options.

The study of anisotropy of acoustic wave propagation in " $Y$-cut LGS/fused silica" layered structure was conducted. Depending on "thickness $\times$ frequency" value, nonlinear material LGS tensors' valuation at computation can both almost completely compensate geometric nonlinearity of the sample and increase the change of the elastic wave phase velocity under condition of external mechanical stress influence.

\section{Acknowledgements}

The research is conducted within the framework of the state task of Ministry of Education and Science of the Russian Federation to Siberian Federal University on R\&D performance in 2014 (Task 3.2534.2014/K) and grant of the President of the Russian Federation for the support of leading scientific schools SS-4828.2012.2.

\section{References}

[1] Sh.Jiang, X.Gong, X.Guo, X.Wang, Potential application of graphene nanomechanical resonator as pressure sensor, Solid State Communications, 193(2014), no. 9, 30-33.

[2] J.Wang, Zh.Huang, H.Duan, Sh.Yu, X.Feng, G.Wang, W.Zhang, T.Wang, Surface stress effect in mechanics of nanostructured materials, Acta Mechanica Solida Sinica, 24(2011), no. $1,52-82$.

[3] K.A.Snook, P.W.Rehrig, W.S.Hackenberger, R.J.Meyer, D.Markley, Tailored single crystal orientations for improved tonpilz transducer performance, Proc. IEEE Ultrasonics Symp. (Vancouver, Canada), 2006, 359-362.

[4] Y.Fan, X.Ji, X.Liu, P.Cai, The nonlinear analysis of elastic wave of piezoelectric crystal plate with perturbation method, Wave Motion, 51(2014), no. 5, 798-803.

[5] F.Kubat, W.Ruile, T.Hesjedal, J.Stotz, U.Rösler, L.M. Reindl, Calculation and experimental verification of the acoustic stress at GHZ frequencies in SAW resonators, IEEE Trans. on Ultrason., Ferroel. and Freq. Control, 51(2004), no. 11, 1437-1448.

[6] Y.Jing, J.Chen, X.Gong, J.Duan, Stress-induced frequency shifts in rotated Y-cut langasite resonators with electrodes considered, IEEE Trans. on Ultrason., Ferroel. and Freq. Control, 54(2007), no. 5, 906-909.

[7] J.A.Kosinski, R.A.Pastore, Jr., J.Yang, X.Yang, J.A.Turner, Stress-induced frequency shifts of degenerate thickness-shear modes in rotated Y-cut quartz resonators, IEEE Trans. on Ultrason., Ferroel. and Freq. Control, 57(2010), no. 8, 1880-1883.

[8] J.A.Kosinski, R.A.Pastore Jr., X.Yang, J.Yang, J.A.Turner, Stress-induced frequency shifts in langasite thickness-mode resonators, IEEE Trans. on Ultrason., Ferroel. and Freq. Control, 59(2009), no. 1, 129-135. 
[9] H.Zhang, J.A.Kosinski, Analysis of contributions of nonlinear material constants to stressinduced velocity shifts of quartz and langasite surface acoustic wave resonators, IEEE Trans. on Ultrason., Ferroel. and Freq. Control, 60(2013), no. 5, 975-985.

[10] S.I.Burkov, B.P.Sorokin, K.S.Aleksandrov, A.A.Karpovich, Reflection and refraction of bulk acoustic waves in piezoelectrics under uniaxial stress, Acoustical Physics, 55(2009), no. 2, $178-185$.

[11] S.I.Burkov, B.P.Sorokin, A.A.Karpovich, K.S.Aleksandrov, Reflection and refraction of bulk acoustic waves in piezoelectric crystals under the action of bias electric field and uniaxial pressure, Proc. IEEE Ultrasonics Symp. (Beijing, China), 2008, 2161-2164.

[12] H.Liu, Z.K.Wang, T.J.Wang, Effect of initial stress on the propagation behavior of Love waves in a layered piezoelectric structure, Int. J. of Solids and Structures, 38(2001), 37-51.

[13] Z.H.Qian, F.Jin, Z.K.Wang, K.Kishimoto, Love waves propagation in a piezoelectric layered structure with initial stresses, Acta Mech., 171(2004), 41-57.

[14] M.El Hakiki, O.Elmazria, F.Bénédic, P.Nicolay, D.Monéger, R.Azouani, Diamond film on Langasite substrate for surface acoustic wave devices operating in high frequency and high temperature, Diamond and Related Materials, 16(2007), no. 4-7, 966-969.

[15] G.Tortissier, L.Blanc, A.Tetelin, J.-L.Lachaud, M.Benoit, V.Conédéra, C.Dejous, D.Rebière, Langasite based surface acoustic wave sensors for high temperature chemical detection in harsh environment: Design of the transducers and packaging, Sensors and Actuators B: Chemical, 156(2011), no. 2, 510-516.

[16] N.Naumenko, L.Solie , Optimal cuts of langasite, $\mathrm{La}_{3} \mathrm{Ga}_{5} \mathrm{SiO}_{14}$ for SAW devices, IEEE Trans. on Ultrason., Ferroel. and Freq. Control, 48(2001), no. 2, 530-537.

[17] P.Zheng, D.W.Greve, I.J.Oppenheim, Langasite surface acoustic wave gas sensors: modeling and verification, IEEE Trans. on Ultrason., Ferroel. and Freq. Control, 60(2013), no. 3, $579-586$.

[18] A.Talbi, F.Sarry, L.Le Brizoual, O.Elmazria, P.Alnot, Sezawa mode SAW pressure sensors based on $\mathrm{ZnO} / \mathrm{Si}$ structure, IEEE Trans. on Ultrason., Ferroel. and Freq. Control, 51(2004), no. $11,1421-1426$.

[19] R. Duhamel, L. Robert, Hongguang Jia, Feng Li, F. Lardet-Vieudrin, J.-F. Manceau, F. Bastien, Sensitivity of a Lamb wave sensor with $2 \mu \mathrm{m}$ AlN membrane, Ultrasonics, 44(Supplement)(2006), e893-e897.

[20] K.S.Aleksandrov, B.P.Sorokin, S.I.Burkov, Effective piezoelectric crystals for acoustoelectronics, piezotechnics and sensors, vol. 2, Novosibirsk, SB RAS Publishing House, 2008 (in Russian).

[21] S.I.Burkov, B.P.Sorokin, A.A.Karpovich, K.S.Aleksandrov, The influence of static homogeneous fields on the properties of SAW in piezoelectrics, Ferroelectrics Letters, 14(1992), no. 5/6, 99-113. 
[22] S.I.Burkov, O.P.Zolotova, B.P.Sorokin, P.P.Turchin, Analysis of the effect of homogeneous mechanical stress on the acoustic wave propagation in the " $\mathrm{La}_{3} \mathrm{Ga}_{5} \mathrm{SiO}_{14}$ / fused silica" piezoelectric layered structures, Ultrasonics, 55(2015), no. 1, 104-112.

[23] B.P. Sorokin, M.P.Zaitseva, Yu.I. Kokorin, S.I,Burkov, B.V. Sobolev, N.A. Chetvergov, Anisotropy of the bulk acoustic wave velocity under the electric field in the sillenite structure piezoelectric crystals, Soviet Phys. Acoust., 32(1986), no. 5, 664-666 (in Russian).

[24] O.P.Zolotova, S.I.Burkov, B.P.Sorokin, A.V.Telichko, Elastic waves in piezoelectric layered structures, J. Siberian Federal University. Mathematics \& Physics, 5(2012), no. 2, 164-186.

[25] K.S.Aleksandrov, B.P.Sorokin, P.P.Turchin, D.A.Glushkov, Non-linear piezoelectricity in $\mathrm{La}_{3} \mathrm{Ga}_{5} \mathrm{SiO}_{14}$ piezoelectric single crystal, Ferroelectrics Letters, 14(1992), no. 5/6, 115-125.

[26] H.J.McSkimin, Measurement of elastic constants at low temperatures by means of ultrasonic waves-data for silicon and germanium single crystals, and for fused silica, J. Appl. Phys., 24(1953), no. 8, 988-997.

[27] I.V.Anisimkin, New type of an acoustic plate mode: quasi-longitudinal normal wave, Ultrasonics, 42(2004), no. 10, 1095-1099.

[28] V.I.Anisimkin, General properties of the Anisimkin Jr plate modes, IEEE Trans. on Ultrason., Ferroel. and Freq. Control, 57(2010), no. 9, 2028-2034.

[29] V.I.Anisimkin, New acoustic plate modes with quasi-linear polarizations orientation, IEEE Trans. on Ultrason., Ferroel. and Freq. Control, 59(2012), no. 10, 2363-2367.

[30] S.I.Burkov, O.P.Zolotova, B.P.Sorokin, P.P.Turchin, Calculation of thermostable directions and the effect of external electric field on the propagation of Lamb and $\mathrm{SH}$ waves in a langasite crystal plate, Acoust. Phys., 58(2012), no. 6, 650-657.

\section{Влияние одноосного механического напряжения на распространение упругой волны в пьезоэлектрических слоистых структурах „,Ү-срез лангасита/плавленый кварц“"}

Сергей И. Бурков

\footnotetext{
Проведено исследование влияния внешнего одноосного механического напряжения на дисперсионные характеристики и анизотропию упругих волн в таких пъезоэлектрических структурах, как "Y -срез $\mathrm{La}_{3} \mathrm{Ga}_{5} \mathrm{SiO}_{14} /$ плавленый квари,". Представлены результаты компъютерного моделирования, выполненного с учетом влияния линейных и нелинейных материальных констант среды. Выполнено сравнение изменений скорости упругой волны под влиянием одноосного напряжения в зависимости от того, учитывался ли при расчетах полный набор нелинейных материальных констант кристаллического слоя + геометрическая нелинейность или только геометрическая нелинейность слоя, вызванная статической деформачией подложки.
}

Ключевые слова: пьезоэлектрическая слоистая структура, волна Рэлея, волна Лява, однородное давление. 